Katarina Melić*

Faculté des Lettres et des Arts

Université de Kragujevac
UDK : 821.133.1-31.09 Sinclair A. DOI: $10.19090 / \mathrm{gff} .2021 .3 .261-275$ originalni naučni rad

\title{
HISTOIRE ET POSTMÉMOIRE : LE RÉCIT FILIAL D’ANNE SINCLAIR
}

Il s'agit d'étudier comment Anne Sinclair, en croisant la mémoire familiale et l'enquête historique, éclaire un des aspects méconnus de la persécution sous l'Occupation : la « rafle des notables » et leur confinement dans le camp de Compiègne. Son grand-père paternel, Léonce Schwartz, fait partie des 743 Français juifs arrêtés en décembre 1941. Tous sont enfermés au camp de Compiègne, sous administration allemande : un vrai camp de concentration nazi d'où partira, en mars 1942, le premier convoi de déportés de France vers Auschwitz (bien avant la Rafle du Vélodrome d'Hiver de juillet 1942). Dans ce récit personnel que l'on peut situer dans le champ d'étude de la postmémoire et sur lequel nous nous appuyons dans notre étude, Anne Sinclair, en reconstituant la coexistence dans ce camp de bourgeois assimilés et de Juifs étrangers, redonne voix et présence aux disparus, et son enquête personnelle devient progressivement une œuvre de mémoire collective. Avec ce récit, A. Sinclair poursuit, après 21, rue La Boétie, son dessein de retracer la mémoire familiale qui a pâti des persécutions à l'égard des Juifs pendant l'Occupation en France.

Mots-clés: postmémoire, récit filial, Shoah, rafle, mémoire individuelle, mémoire collective, $3^{\circ}$ génération.

Éparses, les bribes de mémoires, matérielles ou psychiques, qu'une même histoire peut nous laisser en partage. G.Didi-Huberman

Depuis une quinzaine d'années, une nouvelle génération d'écrivains que l'on nomme les auteurs de la troisième génération s'interroge sur la Shoah alors qu'elle est née bien après. Ces auteurs sont définis comme les petitsenfants des déportés ou des survivants de la Shoah. Placés devant l'absence dans la mémoire familiale, ils veulent savoir et montrer, tout en sachant que

*katarinamelic@yahoo.fr 
cela est impossible. En se penchant sur une mémoire familiale lacunaire et fragmentée, ils sont en quête de sens et d'identité. Derniers liens vivant avec cet événement, ils sont les nouveaux porteurs de la représentation de la Shoah et de la transmission de la mémoire. Ils constituent un pont avec la mémoire future de la Shoah. Ce rapport de distance et de proximité est d'ailleurs une des majeures questions de la création littéraire des écrivains de la troisième génération. L'éloignement de la Shoah soulève des questions d'appropriation et de légitimité. Comment être le narrateur ? Quelle sorte de narrateur ? C'est ce que souligne Daniel Mendelsohn dans son œuvre Disparus dans laquelle il narre l'enquête qu'il a menée pour retrouver les traces de six membres de sa famille disparus en Pologne pendant la Shoah :

Autre façon de dire la même chose, la proximité vous rapproche de ce qui s'est passé, est responsable des faits que nous recueillons, des artefacts que nous possédons, des citations Verbatim des gens que nous enregistrons ; mais la distance est ce qui rend possible l'histoire de ce qui s'est passé, c'est précisément ce qui donne à quelqu'un la liberté d'organiser et de composer ces fragments dans un ensemble plaisant et cohérent - de prendre, par exemple, trois propos séparés, tenus par une personne au cours de trois soirées différentes, et de les enchaîner parce que, de cette façon, ils génèrent un effet dramatique beaucoup plus puissant que si on les trouvait dans trois chapitres successifs d'un livre. (Mendelsohn, 2007 : 511)

Anne Sinclair, journaliste et écrivaine française, fait partie de cette troisième génération qui revient sur ses origines pour finalement mettre en lumière un épisode méconnu de l'Occupation, la rafle des Juifs français et leur internement dans le camp de Compiègne-Royallieu, le deuxième camp le plus important après celui de Drancy. Comme son livre est dédié à ses enfants et à ses petits-enfants, elle se fait le médiateur dans la transmission de l'héritage familial et historique.

Dans notre travail, nous allons avoir recours à deux notions - le récit filial et la postmémoire - dont nous allons souligner les points principaux à la suite. 


\section{LE RÉCIT FILIAL ET LA POSTMÉMOIRE}

\section{Le récit filial}

Sur ce premier point, nous avons fait appel aux études faites par Dominique Viart qui a proposé d'appeler des « récits de filiation » des récits dans lesquels des auteurs cherchent à s'approcher à la fois d'une antériorité et d'une intériorité : ce sont souvent des « récits de filiation » ${ }^{1}$ (Viart, 2005 : 76). Il ne s'agit ni de roman familial, de roman de famille ou de roman de formation. Déjà dans un article publié en 1999, il a montré que la littérature française contemporaine était obsédée par les questions familiales et les problèmes de filiation. Par ceci, il comprend que certains écrivains remplacent l'investigation de leur intériorité par celle de leur antériorité familiale (Viart, 2005 : 79). Les parents, les ancêtres sont l'objet d'une investigation de l'écrivain ou du narrateur dont l'un des buts est une autre connaissance de luimême à travers la connaissance de ceux dont ils sont les descendants. Ces héritiers, tentant de retisser les liens déchirés de l'héritage familial, essaient de (re)composer une identité au détour des ascendants. Pour Viart, le récit filial a pour originalité de substituer au récit plus ou moins chronologique de soi, une enquête sur l'ascendance du sujet. Laurent Demanze souligne à ce sujet que l'auteur d'un récit filial est «[...] à la fois dépossédé ${ }^{2}$ de son inscription généalogique et possédé $e^{3}$ par ces vies antérieures de l'ascendance » (Demanze, 2009 : 12). Dans son article sur les nouveaux modèles d'écriture de l'Histoire (2009), Viart distingue plus loin deux types de textes - le roman archéologique et les fictions de témoignage - qu'il définit explicitement ${ }^{4}$; le premier type est particulièrement répandu parmi les écrivains de la troisième génération de la Shoah. Pour Viart, ce sont des textes qui adoptent une " position d'enquête rétrospective » (Viart, 2009 : 23), de sorte que l'Histoire est abordée « comme une énigme. [...] L'Histoire se dit [...] à partir d'une ignorance de ce qu'elle fut effectivement et ne procède pas du savoir institué » (Viart, 2009: 25). L'enquête autobiographique est doublée d'une enquête

\footnotetext{
${ }^{1}$ Il a premièrement proposé cette notion lors d'un colloque organisé en 1996.

2 En italique dans le texte.

${ }^{3}$ En italique dans le texte.

4 Il relève aussi un certain nombre de procédés dont l'éthique de la restitution et l'écriture du scrupule.
} 
généalogique; le travail sur la mémoire comprend une recherche dans les archives, une collecte de témoignages écrits et oraux afin de faire surgir une mémoire enfouie. Ces textes adoptent la position d'une enquête rétrospective car ils envisagent l'Histoire comme une énigme dans le présent, le narrateur adoptant une " posture d'enquête [...] rétrospective » (Viart, 2009: 23-25). Ces textes sont des enquêtes sur les traces, les zones d'ombre et d'incertitude, les archives. Viart souligne que dans le récit filial/le roman archéologique apparaissent souvent des « traces du passé, lettres, cartes, photographies, objets, reliques et 'documents' de toute nature, lesquels constituent les supports du récit, en forment aussi parfois la matière [...]. » (Viart, 2009: 2627). Les textes de la troisième génération, plus encore que ceux de la deuxième, sont traversés d'archives culturelles (textes littéraires et critiques sur la Seconde Guerre mondiale, photographies, films...), souvent à défaut d'archives familiales. Il s'agit de se plonger dans une mémoire enfouie et de faire ressortir des fantômes/des revenants. Les photographies ont une place de choix dans ces archives familiales dont les récits de filiation dressent l'inventaire.

\section{La postmémoire}

Le second concept auquel nous faisons référence dans cette étude est celui de postmémoire, tel qu'il a été défini dans les travaux de Marianne Hirsch. C'est un terme forgé par Marianne Hirsch dans les années 1990, dans le contexte des études mémorielles américaines, notamment celles portant sur la Shoah. En 1997, elle publie une œuvre qui est devenue incontournable dans les études mémorielles, Family Frames. Photography, Narrative and Postmemory. Cette expression, d'une part, montre la mesure de l'emprise qu'exerce l'histoire de la destruction des Juifs d'Europe sur des individus qui n'ont d'elle qu'une connaissance médiate et distanciée. D'autre part, elle désigne les résultats d'un travail artistique d'appropriation d'une mémoire n'appartenant pas en propre à ceux et celles qui l'adoptent. La notion de «postmémoire » donne une clé qui éclaire la position de ces individus affectés par des événements qui ne sont pas les leurs. Marianne Hirsch définit la postmémoire comme

[...] la relation que la 'génération d'après' entretient avec le traumatisme personnel, collectif et culturel subi par ceux qui l'ont précédée, avec des expériences dont elle ne 'se souvient' que par le biais d'histoires, d'images et de comportements au milieu desquels elle a grandi. Mais ces expériences lui ont été transmises de façon si profonde et avec tant d'émotion qu'elles 
semblent constituer une mémoire en tant que telle. Le rapport de la postmémoire avec le passé est assuré par la médiation non pas de souvenirs, mais de projections, de créations et d'investissment de l'imaginaire. [...] Ces événements sont survenus dans le passé, mais leurs effets continuent dans le présent. C'est là, je le pense, la structure de la postmémoire et le processus propre à sa génération. (Hirsch, $2008: 106-107)^{5}$

La postmémoire se distingue de la mémoire par la distance générationnelle et de l'histoire par une profonde connexion personnelle. C'est une forme très particulière de mémoire parce que le lien qu'elle entretient avec son objet et sa source n'est pas celui des souvenirs, mais celui de l'imaginaire et de la création. Il s'agit d'une forme indirecte de mémoire qui ne peut se réaliser que dans une dimension imaginaire. C'est un terme qui exclut ainsi le témoin (pas de "souvenirs» mais un «investissement imaginaire»), tout en faisant de l'auteur d'un récit postmémoriel, un témoin second ou par procuration. Pour Évelyne Ledoux-Beaugrand, la postmémoire désigne surtout « [...] une connexion au passé établie par une forme d'appropriation créative qui s'efforce de réactiver et de réincorporer des structures mémorielles distantes. » (Ledoux-Beaugrand, 2013: 149). Selon elle, le concept de postmémoire

[...] consiste à adopter un point de vue ancré dans le présent et à ainsi aborder le génocide à partir de ses traces encore repérables dans l'espace et le temps contemporains. Cette littérature postmémorielle s'emploie moins à jeter un regard sur le passé qu'à tirer au jour les restes de ce passé dont est pétri le présent. (Ledoux-Beaugrand, $2013: 146$ )

Anne Sinclair fait partie de cette génération d'écrivains, communément appelée la « troisième génération », qui n’a « de la Shoah qu'une connaissance médiate, portée notamment par ses représentations littéraires et cinématographiques » (Ledoux-Beaugrand, 2013 : 146). Comme le rappelait Marianne Hirsch,

La deuxième génération s'est comportée en gardien, déterminant les formes de transmission qui étaient valables et celles qui ne l'étaient pas. Mais il nous faut maintenant prendre conscience que nous devons nous-mêmes transmettre l'histoire à la troisième génération et la mettre à la disposition

5 Notre traduction. 
d'autres personnes, de telle sorte qu'elles puissent la relier à leurs propres histoires, qui sont très diverses. (Hirsch, $2013: 6$ )

Ceci dit, dans le cas des écrits d'Anne Sinclair, nous retrouvons ce que Marianne Hirsch nomme « la postmémoire familiale », pour la distinguer de " la postmémoire affiliative », qui n'est pas éprouvée par un descendant direct du traumatisme passé, c'est-à-dire d'un récit post-mémoriel où, d'après Ledoux-Beaugrand, "La postmémoire nomme à la fois une structure de transmission trans et intergénérationelle et la posture d'une génération face à des événements traumatiques éloignés d'elle dans le temps et parfois aussi dans l'espace et dans la langue. » (Ledoux-Beaugrand, 2013: 148). La recherche et la place des documents familiaux et administratifs, leur place, l'enquête et la description de ses étapes sont des étapes incontournables dans la démarche postmémorielle, ce que le récit filial à l'étude inclue à la lettre.

\section{HISTOIRE ET POSTMÉMOIRE}

\section{1, rue La Boétie}

La première étape dans la constitution d'un récit filial et postmémoriel, Anne Sinclair la pose en publiant en 2012 chez Grasset, 21, rue la Boétie. Elle y narre un épisode de sa famille à laquelle elle ne s'est pourtant pas intéressée dans sa jeunesse: « Je suis la petite-fille d'un monsieur qui s'appelait Paul Rosenberg et qui habitait à Paris, au 21 de la rue la Boétie » (Sinclair, 2012 : 20).

Avec cette dernière phrase du prologue de son livre, Anne Sinclair se tourne vers l'Histoire, son histoire, et celle de son grand-père, Paul Rosenberg (1881-1959), un des marchands d'art parisiens les plus influents de l'entredeux-guerres. Passionné par l'art, propriétaire d'une galerie d'art au 21, rue La Boétie $^{6}$, il est le premier à signer en 1918 avec Pablo Picasso ${ }^{7}$ un contrat lui garantissant l'exclusivité de sa production. Pour sceller cette union - et aussi le

6 Ironie de l'histoire, la galerie de la rue La Boétie va accueillir les expositions antisémites de l'Institut d'Étude des Questions Juives jusqu'à la Libération.

7 Une profonde amitié s'était tissée entre Picasso que Rosenberg appelait Pic, et Rosenberg que Picasso appelait Rosy de sorte que Picasso s'est installé rue La Boétie, $\mathrm{au}$ numéro 23, tout près de la galerie Rosenberg, pour pouvoir dialoguer à l'envi avec son marchand. 
début d'une amitié qui durera quarante années, le peintre espagnol offre à la famille Rosenberg, un tableau Portrait de Mme Rosenberg et sa fille, un portrait de Mathilde avec sa fille sur ses genoux - la mère d'Anne Sinclair. Le début de la Deuxième Guerre mondiale voit arriver l'occupation de Paris, les premières mesures anti-juives, la spoliation et le pillage des musées, galeries et marchands d'art. Les établissements Juifs sont principalement pris pour cible Bernheim-Jeune, Alphonse Kahn, Paul Rosenberg, Jacques Seligmann, les frères Wildenstein. L'intégralité de leurs collections est confisquée et envoyée vers l'Allemagne. Les Rosenberg fuient Paris et se réfugient pour un temps dans la région de Bordeaux ; Rosenberg parvient à faire revenir de Paris cent soixantedeux tableaux de Vincent Van Gogh, Camille Corot, Eugène Delacroix, Maurice Utrillo, etc. et les cache dans un coffre d'une banque de Libourne. En septembre 1941, les nazis, informés, font ouvrir le coffre et confisquent l'intégralité de son contenu qu'ils font envoyer à Hermann Göring qui veut devenir un des plus grands collectionneurs d'art de l'époque ${ }^{8}$. Déchu de sa nationalité française, Rosenberg fuit avec sa famille à travers l'Espagne, le Portugal pour arriver aux États-Unis, avec l'aide d'Alfred Barr, alors conservateur au MoMA, qui va convaincre les autorités américaines d'accorder un visa aux Rosenberg. En signe de reconnaissance pour cette aide inespérée, Paul Rosenberg enrichit les collections des plus prestigieux musées des États-Unis. Après la guerre, Paul Rosenberg et ses proches engagent alors la lutte pour la restitution des oeuvres pillées et reconstituent à peu près la collection.

\section{La rafle des notables}

Anne Sinclair s'était attachée au versant maternel de sa famille, dans 21, rue La Boétie, à travers la figure de son grand-père. Elle se tourne vers le versant paternel de sa famille, à travers la figure de son autre grand-père, Léonce Schwartz, dans La Rafle des notables, publié en 2020. Si les deux livres ne se ressemblent pas du tout, ils ont une base commune : la quête. Anne Sinclair y raconte la " rafle des notables », récit où la mémoire familiale rejoint l'enquête historique, et éclaire certains aspects méconnus de la persécution des juifs français.

${ }^{8}$ Voir le livre d'Emmanuelle Polack (2019), Le marché de l'art sous l'Occupation. Paris : Tallandier. 
Le 12 décembre 1941, les Allemands arrêtent 743 Juifs français, appartenant à une population privilégiée. Et pour arriver au chiffre de 1000 que s'était fixée la Gestapo, ils y ajoutent 300 juifs étrangers, déjà prisonniers à Drancy. Ils sont internés au camp de concentration nazi de Compiègne ${ }^{9}$, sous administration allemande, dans des conditions inhumaines. Le premier convoi de déportés de France vers Auschwitz part le 27 mars 1942 du camp de Compiègne. Le grand-père paternel d'Anne Sinclair, Léonce Schwartz, commerçant en gros de dentelles prospère, établi dans les beaux quartiers de la capitale, il est réveillé au petit matin par deux soldats allemands et deux policiers français. Il a été arrêté lors de la « rafle des notables » et interné au camp de Compiègne. Il fait partie des 743 Français juifs arrêtés ce jour-là, bourgeois et intégrés comme lui :

Selon le décompte de Serge Klarsfeld, ils étaient ce matin-là 390 commerçants et directeurs de société, 322 artisans, 91 ingénieurs, 63 médecins et dentistes, 33 pharmaciens et chimistes, 31 étudiants, 27 professions libérales, 16 avocats, 11 professeurs et 53 sans profession. (Sinclair, $2020: 24$ )

Parmi eux se trouvaient des intellectuels, des magistrats, des médecins, des écrivains ${ }^{10}$, des scientifiques, des chefs d'entreprise :

Pour atteindre le chiffre de 743 Juifs français, considérés comme 'notables', autrement dit influents ('einflussreiche Juden'). René Blum, directeur des Ballets de Monte-Carlo, frère de Léon, est parmi eux. Ainsi que Roger Masse, polytechnicien et militaire dans l'armée française. Son frère, Pierre Masse, éminent juriste, sénateur, ancien membre du gouvernement Clemenceau, arrêté depuis août 1941, le rejoindra le lendemain. Jacques Debré, ingénieur, frère du grand médecin le professeur Robert Debré, est présent dans le manège, comme $\mathrm{M}$. Ullmo le célèbre avocat. Ainsi que Robert Dreyfus, conseiller à la Cour de cassation, Édouard Laemlé, président de chambre à la cour d'appel de Paris, ou Georges Wellers, déjà mentionné, futur historien du nazisme, qui sera déporté à Auschwitz et survécut. Jean-Jacques Bernard, fils de Tristan Bernard, côtoie Maurice Goudeket, le mari de Colette. C'est la particularité de cette rafle (où 13 des hommes arrêtés sont polytechniciens et 55 portent l'insigne de la Légion d'honneur) de rassembler une population

9 «Les Allemands voulurent-ils, par symbolique historique, installer un camp à cet endroit en souvenir de l'humiliant armistice signé le 11 novembre 1918 dans un wagon à Rethondes, sur la commune de Compiègne ? » (Sinclair, 2020 : 21-22)

${ }^{10}$ Robert Desnos est passé par ce camp. 
socialement homogène. Les professions libérales sont sur-représentées. Ainsi que des commerçants, comme mon grand-père. (Sinclair, 2020 : 17)

Extrêmement affaibli, le grand-père, chevalier lui-aussi de la Légion d'honneur, a été transféré à l'hôpital du Val-de-Grâce :

Pourtant, les 18 et 20 décembre, 73 détenus considérés comme de très grands malades et 20 hommes de plus de 65 ans furent libérés, ainsi que 15 autres sur ordre spécial. Ces libérations groupées furent l'exception pendant trois mois. Il y eut ici ou là des libérations individuelles, souvent d'hommes en si mauvais état que les Allemands préféraient les voir mourir à l'hôpital. La mort dans ce camp de 'notables' faisait mauvais effet auprès de Vichy. Et à l'époque, cela comptait encore un peu. (Sinclair, $2020: 26$ )

Son épouse réussit à le faire sortir de l'hôpital. Ils se cachent jusqu'à la Libération. Léonce Schwartz meurt en mai 1945, dans sa maison et dans son lit, des suites de son internement à Compiègne. Anne Sinclair souhaitait raconter la vie de son grand-père paternel mais elle restitue, à travers lui, les morts et les vivants du camp de Compiègne. Elle part d'un homme pour aller vers les hommes. Rechercher l'histoire d'un aïeul et en découvrir des centaines d'autres.

L'épisode de la rafle des notables et l'existence du camp de Compiègne sont longtemps restés méconnus du grand public. $\mathrm{Ni}$ historienne, ni chercheuse, Sinclair mène une enquête sur les circonstances de l'arrestation de son grand-père, mais cette enquête devient vite bien plus qu'une histoire familiale: en cherchant des documents sur le camp de Compiègne, elle s'aperçoit qu'elle n'est pas la seule à ne jamais en avoir entendu parler.

À la faveur de cette enquête familiale et historique, Anne Sinclair reconstitue le destin tragique des internés de ce camp. L'enquête familiale sur le destin de son grand-père devient peu à peu une enquête historique sur la tragédie de Compiègne. Sinclair découvre que cette rafle des notables de décembre 1941 est la troisième (après celles de mai 1941 puis d'août 1941), et donc, six mois avant la rafle du Vel d'Hiv (juillet 1942) connue de tous. Elle a été la première qui a fourni un «contingent» de Juifs de France en direction des camps d'extermination (Auschwitz principalement). Ce convoi est parti de Compiègne le 27 mars 1942. Très peu d'entre eux revinrent. Ensuite, c'est le ciblage par les nazis, des "notables», autrement dit, des bourgeois assimilés depuis bien longtemps, et donc une population qu'ils ont voulue homogène de « juifs influents » selon leur formule. 
Le lecteur découvre les conditions déplorables du camp à 70 kilomètres de Paris, où régnaient l'élimination par la faim, le froid, la maladie, la saleté, les conditions terribles d'un hiver à $-20^{\circ}$. Beaucoup y sont morts de faim, beaucoup ont perdu 20 à 30 kilogrammes en 3 mois, beaucoup ont eu les pieds gelés à rester debout des heures dans la neige, beaucoup sont morts de dysenterie, etc... Y règnent la famine, le froid, la saleté, la gangrène. C'est un lieu de souffrance dont le but est l'extermination. Sinclair informe le lecteur que sans la solidarité des autres camps de prisonniers qui existaient dans ce périmètre de Compiègne (notamment le camp des communistes et celui des Russes, un peu mieux lotis), les morts dans la partie du camp, appelée « camp des Juifs » (que les Allemands voulaient être pire que les autres), auraient été encore plus nombreux avant même leur déportation en 1942 :

Ceux qui sortirent de Compiègne vers la liberté, l'hôpital, la clandestinité ou la déportation, repartiront en ayant perdu 25 à 30 kilos en trois mois. La malnutrition, le froid, la maladie ont commencé de transformer les prisonniers en silhouettes fantomatiques. Leur température était souvent proche de 35 degrés ou de 34 degrés, et avec de la fièvre, montait à 36,5 degrés. À l'inverse, quand elle descendait à 33,5 degrés, la fin était proche. (Sinclair, $2020: 43$ )

Anne Sinclair relate aussi les différences entre les Juifs privilégiés se sentant français et les Juifs étrangers habitués aux persécutions. Elle évoque les figures remarquables de ce camp, comme le dentiste Benjamin Schatzman, pour leur redonner un souffle de vie. À l'intérieur du camp, la vie intellectuelle a sauvé bien des internés de la folie. Ils récitaient des poèmes, donnaient des conférences :

Blaise Pascal, l'art, l'agriculture, le pétrole, la comptabilité, les possibilités de la science, firent partie des sujets de conférences très suivies. L'ingénieur Louis Engelmann, le voisin de Léonce, donna une leçon sur l'électricité ; René Blum sur Alphonse Allais, Tristan Bernard et Courteline ; Jean-Jacques Bernard sur le théâtre ou sur la poésie française au Moyen Âge ; Georges Ullmo sur les grands procès d'assises ; Jacques Ancel sur l'idée de nation. Deux sujets étaient proscrits : l'Allemagne, par crainte des mouchards, et le problème juif. (Sinclair, $2020: 39$ )

Aujourd'hui, les noms des internés du camp de Compiègne sont gravés dans le verre à l'entrée du mémorial. La petite-fille voulait que le nom de son grandpère y figure. 


\section{L'incuriosité et l'impouvoir}

L'écrivaine-journaliste souligne bien de fois l'incuriosité de sa jeunesse qu'elle regrette aujourd'hui :

Cette histoire me hante depuis l'enfance. Et pourtant, si les sagas familiales interpellent ceux qui avancent en âge, la mienne ne m'a d'abord intéressée que de loin, l'immédiateté ayant eu longtemps, dans ma vie de journaliste, plus d'attrait que les histoires du passé. Néanmoins, ce chapitre-là continue de me poursuivre. Pour le côté romanesque que je n'ai pas pu élucider, pour les questions que je n'ai pas posées, pour les détails que je n'ai pas demandés? (Sinclair, $2020: 4$ )

Me manquent donc les réponses aux questions que je n'ai pas posées et qui me taraudent aujourd'hui. Bien tard. Trop tard. (Sinclair, $2020: 53$ )

Son besoin de savoir a suscité une enquête. Elle a posé des questions aux autres, mais pas aux siens :

Je rôdais autour de l'autre, celle de ma famille paternelle, sans y pénétrer. Et comme toujours, quand les témoins disparaissent, quand plus personne n'est là pour affirmer ou raconter, l'urgence s'impose car les bribes de mémoire qui nous sont parvenues s'évanouiront après nous. (Sinclair, $2020: 4$ ).

Les proches meurent en emportant avec eux tout ce qu'on ne leur a pas dit et tout ce qu'ils n'ont pas dit :

On n'interrogeait pas les grands quand on était enfant. Parce que si les confidences ne nous étaient pas faites, il nous semblait indécent de les provoquer. Parce que cela nous pesait, aussi. Écouter les histoires des anciens de la famille, dont on se moquait bien, auxquelles on ne comprenait pas tout, nous embarrassait et empiétait sur les heures de jeux. Pour autant, j'aurais pu, plus tard, chercher à en savoir davantage. Pourquoi ne l'ai-je pas fait, et pourquoi mon père ne m'a-t-il rien raconté, alors qu'assez tôt j'adorais qu'il me lise son journal de guerre dont il expurgeait les pages qui ne regardaient pas l'adolescente que j'étais? (Sinclair, $2020: 4$ )

Donc peu d'éléments pour écrire une histoire familiale. Pas question non plus d'en faire un roman, car j'aurais l'impression de trahir le récit si douloureux qu'ont livré les quelques survivants. (Sinclair, $2020: 7$ ) 
Au départ, il y a eu une surprise. Depuis toujours, Anne Sinclair pensait que son grand-père paternel, Léonce Schwartz, avait été enfermé au camp de Drancy, et qu'il s'en était échappé grâce à son épouse :

Quand j'ai commencé mes recherches sur Léonce Schwartz, le père de mon père, j'espérais dénicher des pistes : sur son arrestation - que j'ai cru effectuée par la police française -, son incarcération - dont j'étais persuadée qu'elle avait eu lieu à Drancy. Et puis, je pensais élucider ce que racontait la légende familiale, une évasion rocambolesque hors du camp, grâce à l'audace de ma grand-mère, déguisée en infirmière, subtilisant une ambulance de la CroixRouge, se faufilant dans le camp, mon grand-père dont elle aurait sauvé la vie assis sur le siège arrière, lui épargnant ainsi de justesse la déportation ! Je présumais trouver des documents, des lettres, ainsi que des traces de leur cachette durant les années qui suivirent. (Sinclair, $2020: 5$ )

Sans aucunes archives, aucune base de départ et de travail - elle se lance dans son entreprise et réalise très vite la naïveté et la presque impossiblité de son but. Elle reconnaît devoir une dette à Serge Klarsfeld et à ses travaux qui ont servi de piste dans ses recherches :

Ce livre n'existerait pas sans les travaux de Serge Klarsfeld et les publications qu'il a voulues éditées par le Mémorial de la Shoah et auxquelles je fais référence tout au long du livre : journaux des internés de Compiègne, ceux qui ont survécu, ceux qui sont revenus des camps, ou ceux qui sont morts et qui ont jeté, par la fenêtre du train qui les emmenait, des petits bouts de papier... Je n'ai pas fait de travail scientifique, mais je me suis vue comme une sorte de relais d'une histoire que j'ai voulu faire connaître au plus grand nombre. C'est à ce moment-là que Karine Taïeb, responsable du service Archives du Mémorial de la Shoah, m'a dit qu'elle ne trouvait pas de traces de mon grandpère à Drancy, mais à Compiègne : [...] et je me nourrirais de ma quête et ses apories pour en faire un récit littéraire, dans le sillage de Dora Bruder de Patrick Modiano. (Sinclair, $2020: 5$ )

C'est Karine Taïeb qui retrouve des traces de son grand-père à Compiègne, et non pas à Drancy. C'est elle encore qui lui apprend sa date d'arrestation dans ce qui a été la "rafle des notables ». Elle insiste sur le fait de ne pas être une historienne, de ne pas avoir fait de travail scientifique - elle se voit comme une sorte de médiateur d'une histoire qu'elle veut faire connaître au grand public. Sinclair ne peut donner des résultats espérés de son enquête, mais trace l'enquête elle-même: ses modalités, ses buts, ses efforts, ses difficultés, le doute, le malaise. Elle souligne bien qu'au départ de sa quête, il s'agit d'une 
réflexion personnelle. Pendant bien des années, elle s'est contentée d'un récit très général sur son grand-père. De ce qui devait être un livre sur son grandpère, Sinclair écrit sur une histoire collective, un drame collectif :

Essayer de redonner un peu de chair aux disparus est devenu pour moi une obsession. (Sinclair, $2020: 7$ )

Je voudrais simplement rendre hommage aux hommes qui ont souffert aux côtés de mon grand-père que je n'ai pas connu et peut-être ainsi atténuer la culpabilité de n'avoir pas tenté plus tôt de démêler les fils de cette histoire.

(Sinclair, $2020: 7$ )

Sinclair s'inscrit bien dans l'aire des études de la postmémoire : la place des documents familiaux et de la recherche de témoignages s'avère cruciale dans la démarche post-mémorielle. Marianne Hirsch a fortement souligné le rôle des photographies et des archives dans l'activation de la mémoire. Évelyne Ledoux-Beaugrand est précise à ce sujet :

[...] le recours à des sources intimes et familiales (photographies, lettres, journaux intimes, correspondances, histoires de famille transmises par bribes) relève généralement de la nécessité dans les œuvres postmémorielles qui cherchent à réactiver la mémoire d'anonymes disparus dans les camps nazis (Ledoux-Beaugrand, $2013:$ 149).

L'enquête familiale devient petit à petit une enquête historique sur la tragédie historique du camp de Compiègne - Léonce passe comme une ombre et un témoin invisible du récit. Anne Sinclair n'a pas retrouvé de nouveaux éléments sur l'arrestation, la détention et la libération de Léonce Schwartz. Elle n'est pas parvenue à faire revivre l'histoire familiale, ni à combler le vide de la mémoire. Le récit filial doit exhumer une transmission défaite, donner la parole à une ascendance condamnée au silence. La postmémoire familiale est souvent vécue comme une obligation, voire une responsabilité de mettre en récit la mémoire de la Shoah par une génération qui a la conscience d'appartenir à une génération qui verra mourir le dernier des survivants de la Shoah. En quête d'une histoire, Sinclair en trouve des centaines d'autres, bien plus tragiques. S'il s'agit d'ajouter une page individuelle à l'histoire, ce récit participe à la mémoire collective :

Ce tourment, passager clandestin de ma propre mémoire, de ma propre histoire, j'espère pouvoir, en l'écrivant, le déposer comme un fardeau intime devenu mémoire collective. (Sinclair, 2020 : 8) 
Katarina Melić

HISTORY AND MEMORY: THE FILIAL NARRATIVE OF ANNE SINCLAIR

\section{Summary}

Our aim was to study how, according to the operative concept of "postmemory" defined by Marianne Hirsch, Anne Sinclair, by crossing the family memory and the historical investigation, shed light on one of the unknown aspects of the persecution under the Occupation in France: the "roundup of the notables" and their confinement in the camp of Compiègne nearby Paris. With this story, A. Sinclair continued, after having previously paid tribute to her maternal grand-father in 21, rue La Boétie, her aim to retrace the family memory which suffered from the persecutions against the Jews during the Occupation in France. Her paternal grandfather, Léonce Schwartz, was one of the 743 French Jews arrested in December 1941. All were detained in the Compiègne camp, under German administration: a real Nazi concentration camp from which the first convoy of deportees left from France for Auschwitz in March 1942. In this personal story at the beginning, Anne Sinclair, by reconstituting the coexistence in this camp of assimilated bourgeois and foreign Jews, gave voice and presence to the disappeared, and her personal investigation became gradually a work of collective memory. In our analysis, our first step was to outline, shortly and precisely, the concept of filial narrative and postmemory whose we applied further in our work.

Key words: Postmemory, Filial Narrative, Shoah, Raid, Individual Memory, Collective Memory, Third Generation.

\section{RÉFÉRENCES BIBLIOGRAPHIQUES}

Aarons, V.-Berger A. L. (2017). Third-Generation Holocaust Representation. Trauma, History and Memory. Evanston : Northwestern University Press.

Bouju, E. (2006). La Transcription de l'histoire. Essai sur le roman européen de la fin $d u$ XXe siècle. Rennes : PUR.

Demanze, L. (2009). Les possédés et les dépossédés. Études françaises, 45, 3, 11-23.

Didi-Huberman, G. (2020). Éparses. Voyage dans les papiers du ghetto de Varsovie. Paris : Minuit.

Hirsch, M. (1997). Family Frames: Photography, Narrative and Postmemory. Cambridge, MA : Harvard University Press.

Hirsch, M. (2008). The Generation of Postmemory. Poetics today, 29:1, 104123. 
Hirsch, M. (2013). Postmémoire. Artabsolument, avril 2013, numéro spécial, 16.

Ledoux-Beaugrand, E. (2013). Les restes d'Auschwitz : intertextualité et postmémoire dans Jan Karski de Yannick Haenel et C'est maintenant du passé de Marianne Rubinstein. Études françaises, 49/2, 145-162.

Leichter-Flack, F. (2014). Second Generation, Third Generation and State Political Postmemory: The Holocaust and Its Literary Effects in Contemporary France. Journal of Literature and Trauma, 4 (1), 67-77.

Mendelsohn, D. (2007). Les Disparus. Paris : Flammarion.

Polack, E. (2019). Le marché de l'art sous l'Occupation 1940-1944. Paris: Tallandier.

Rothberg, M. (2009). Multidirectional Memory: Remembering the Holocaust in the Age of Decolonization. Stanford : Stanford University Press.

Sinclair, A. (2012). 21, rue La Boétie. Paris : Grasset.

Sinclair, A. (2020). La rafle des notables. Paris : Grasset.

Viart, D. (1999). Filiations littéraires. In: Baetens, J.-Viart, D. (éds.) (1999). États du roman contemporain. Écritures contemporaines 2. Caen : Minard. 115-139.

Viart, D.-Vercier, B. (2005). La Littérature française au présent. Héritage, modernité, mutations. Paris : Bordas.

Viart, D. (2009). Nouveaux modèles de représentation de l'Histoire en littérature contemporaine, in : Viart, D. (éd.) (2009). Nouvelles écritures littéraires de l'Histoire, tome X, Écritures contemporaines. Caen : Minard. 11-39.

Schulte Nordholt, A. (2008). Perec, Modiano, Raczymow. La Génération d'après et la mémoire de la Shoah. Amsterdam : Rodopi. 
